\title{
Distributive Opportunistic Spectrum Access for Cognitive Radio using Correlated Equilibrium and No-regret Learning
}

\author{
Zhu Han*, Charles Pandana, and K. J. Ray Liu \\ * Electrical and Computer Engineering Department, Boise State University, USA \\ Electrical and Computer Engineering Department, University of Maryland, College Park, USA
}

\begin{abstract}
Cognitive radio has been proposed as a novel approach for improving the utilization of the precious limited radio resources by dynamically accessing the spectrum. One of the major design challenges is to coordinate and cooperate in accessing the spectrum opportunistically among multiple distributive users with only local information. In this paper, we propose a game theoretical approach with a new solution concept, the correlated equilibrium, which is better compared to the noncooperative Nash equilibrium in terms of spectrum utilization efficiency and fairness among the distributive users. To achieve this correlated equilibrium, we construct an adaptive algorithm based on no-regret learning that guarantees convergence. From the simulation results, the optimal correlated equilibria achieve better fairness and 5\% 15\% performance gain, compared to the Nash equilibria.
\end{abstract}

\section{INTRODUCTION}

With advances of wireless technologies, more and more bandwidths have been licensed for different wireless standards. Due to the ever increasing demand in wireless applications, the electromagnetic radio spectrum has become a very precious natural resource. To overcome this problem, the Federal Communications Commission (FCC) initiated a study of this issue in November 2002. The study revealed that more than $70 \%$ of the spectrum is unutilized in most area. This under-utilization of electromagnetic spectrum leads to the term spectrum holes [1], [2]. Formally, the spectrum hole is defined as a band of frequencies assigned to a primary user, but, at a particular time and specific geographic location, the band is not being utilized by that user. The efficiency of spectrum utilization can be improved significantly by having a secondary user to access a spectrum hole which is unoccupied by the primary user at the right place and at the right time. Cognitive radio, which can sense the spectrum utilization of the primary user and opportunistically access the spectral holes, has been proposed to promote the efficient spectrum usage [1], [2]. Some major problems in cognitive radio are the opportunistic spectrum access, coordination among secondary users, and cognitive MAC.

Recent literature targeting the above problems is as follows. In [3], the authors study metrics to quantify the characteristics of opportunistic spectrum access, namely equivalent non-opportunistic bandwidth and space-bandwidth product. Sensing-based opportunistic channel access is proposed in [4]. In this work, they address whether an accessible channel is a good opportunity for a secondary user. Decentralized cognitive medium access based on partial observable Markov decision process (POMDP) is presented in [5]. Spectrum sharing with distributed interference compensation by means of pricing is presented in [6]. Finally, the rule-based device-centric spectrum management scheme is proposed in [7].
Most of the existing works assume the available signaling among cognitive users to coordinate the spectrum usage. To achieve the distributed implementation, game theory is a natural, flexible, and rich tool to study how the autonomous nodes interact and cooperate with each other. There are many applications of game theory in wireless networking, such as noncooperative power control game [8], cooperative game for OFDMA [9], self-learning repeated game in ad hoc networks [10], and a general survey [11]. In cognitive radio literature, spectrum sharing for unlicensed band using the one shot game and repeated game is proposed in [12]. In [13], the resource allocation for secondary users is formulated as potential game. A survey is studied in [14].

In this paper, we study the behavior of individual distributed secondary user to control its rate when the prime user is absent. Each secondary user seeks to maximize its rates over different channels. However, excessive transmissions can cause the collisions with the other second users. The collisions reduce not only the system throughput but also individual performances. From the game theory perspective, we propose a distributed protocol based on an adaptive learning algorithm for multiple secondary users using only local information. We study a new concept, correlated equilibrium, which is a better solution compared to the non-cooperative Nash Equilibrium in terms of spectrum utilization efficiency and fairness among the users. Using the correlated equilibrium concept, the distributive users adjust their transmission probabilities over the available channels, so that the collisions are avoided and the users' benefits are optimized. We exhibit the adaptive regret-matching (no-regret) algorithm to learn the correlated equilibrium in a distributed manner. We show that the proposed learning algorithm converges to a set of correlated equilibria with probability one. From the simulations, the optimal correlated equilibria achieve better fairness and 5\% 15\% better performances, compared to the Nash equilibrium.

The rest of this paper is organized as follows: In Section II, we present the system model and utility function. In Section III, we study the correlated equilibrium. Then, we construct an adaptive no-regret learning algorithm and show that the algorithm converges to a set of correlated equilibria. Simulation results are shown in Section IV and finally conclusions are drawn in Section V.

\section{SySTEM MODEL}

In this section, we consider the general models for dynamic opportunistic spectrum access for cognitive radio, in which there exist several primary users with a set of available channels and a large number of secondary users. The primary users are 
the rightful owners and have strict priority on the spectrum access. The secondary users are equipped with the spectrumagile devices, which are used by the secondary users to sense the environment (spectrum usage of the primary users) and adapt the suitable frequency, power, and transmission scheme. The secondary users can opportunistically access the spectrum while the primary users are idle. The primary users are the legacy users that communicate in the traditional way and do not retrofit the secondary users. Hence, the channel availability of secondary users inherently depends on the activities of the primary users. Moreover, the secondary users have to compete for the idle channels among the interfering secondary users. If collisions occur, there are some penalties in the forms of packet loss and power waste. This is the major focus of this paper.

\section{A. Network Model}

We consider there are $N$ channels in the wireless networks. Without loss of generality, each channel has a unit bandwidth. These channels are shared among $M$ primary users and $K$ secondary users seeking for channel access opportunistically.

For adjacent secondary users, they can interference with each others. We use interference matrix $\mathbf{L}$ to depict the interference graph. The interference matrix has the dimension of $K$ by $K$, and its elements are defined as

$$
\mathbf{L}_{i j}= \begin{cases}1, & \text { if } i \text { and } j \text { interfere with each other, } \\ 0, & \text { otherwise. }\end{cases}
$$

The interference matrix depends on the relative location of the secondary users.

Next, we define channel availability matrix as a $K$ by $N$ matrix, $\mathbf{A}(t)$. Each user can transmit over a specific channel with a set of different rates. The elements of the matrix is defined as

$$
\mathbf{A}_{i n}(t)= \begin{cases}1, & \text { if channel } n \text { is available for secondary } \\ & \text { user } i \text { at time } t \\ 0, & \text { otherwise. }\end{cases}
$$

We note that the channel availability matrix $\mathbf{A}(t)$ varies over time. This matrix is the result of sensing task done by secondary users, and depends on the primary users' traffic, relative location between the secondary users and the primary users. Notice that each individual user only knows its corresponding row of matrix $\mathbf{L}$ and $\mathbf{A}(t)$.

We illustrate an instantaneous example of the channel availability to the secondary users using the footprint abstraction [3] as the space occupancy of the primary users in Figure 1. There are 4 channels (namely channel $A, B, C$, and $D$ ), 3 sets of primary users, and 5 secondary users. The channel availability of secondary users are also determined by its location with respect to the primary users ${ }^{1}$. From this figure, the primary user I, II, and III occupy channel A, B, and $(\mathrm{A}, \mathrm{C})$, respectively, hence, the secondary users 1 to 5 can only opportunistically access channel (B,C,D), (A,C,D), (D), (B,D), and $(\mathrm{B}, \mathrm{D})$, respectively. Furthermore, two secondary users that interfere with each other are connected with an edge. Hence, they conflict with each other on using the same spectrum.

\footnotetext{
${ }^{1}$ In this example, the interference matrix is assumed symmetric
}

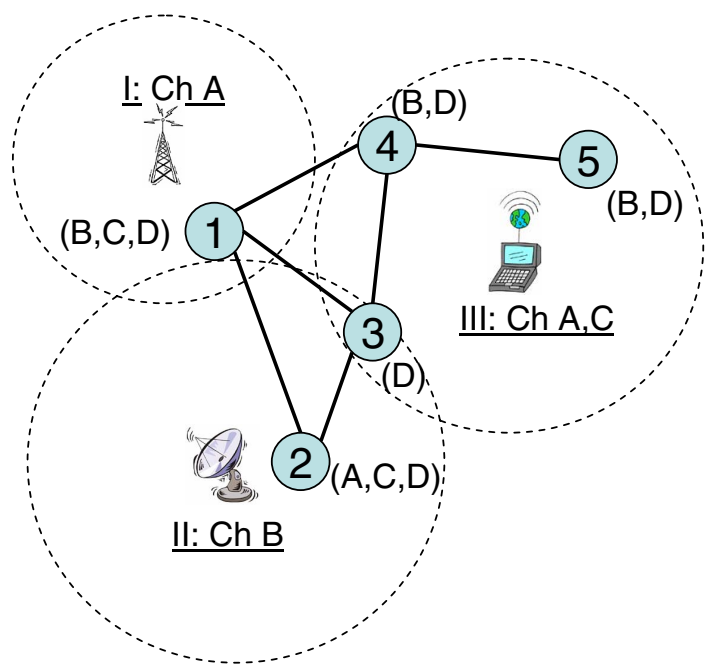

Fig. 1: Illustration of channel availability for secondary users

\section{B. Utility Function}

Define the set of secondary user $i$ as $I$ which is the finite set $\{1,2, \ldots, K\}$. For each available channel, a secondary user can select $L+1$ discrete rates $\Upsilon=\left\{0, v_{1}, \ldots, v_{L}\right\}$. The strategy space $\Omega_{i}$ for secondary user $i$ is on the available channels and can be denoted as

$$
\Omega_{i}=\prod_{n=1}^{N} \Upsilon^{\mathbf{A}_{i n}}
$$

The action of user is $r_{i}^{n}=v_{l}$ representing user $i$ occupies channel $n$ by rate $v_{l}$. We define the strategy profile $\mathbf{r}^{n}=$ $\left(r_{1}^{n}, r_{2}^{n}, \ldots, r_{K}^{n}\right)^{\prime}$, and we define $\mathbf{r}_{-i}^{n}$ as the strategies of user $i$ 's opponents (interference neighbors defined in $\mathbf{L}$ ) for channel $n$. We also define $\mathbf{r}_{i}=\left(r_{i}^{1}, \ldots, r_{i}^{N}\right)^{\prime}$ as the action of users over all channels, and $\mathbf{r}_{-i}$ as the user $i$ 's opponents' actions.

The utility function $U_{i}$ measures the outcome of secondary user $i$ for each strategy profile $\mathbf{r}^{1}, \ldots, \mathbf{r}^{N}$ over different channels. In this paper, we define the utility function as the maximum achievable rate for the secondary users over all the available channels as:

$$
U_{i}=\sum_{n=1}^{N} \mathbf{A}_{i n} R_{i}\left(r_{i}^{n}, \mathbf{r}_{-i}^{n}\right),
$$

where $R_{i}\left(r_{i}^{n}, \mathbf{r}_{-i}^{n}\right)$ is the outcome of resource competition for user $i$ and the other users. Notice that the utility function represents the maximum achievable rate. In practice, the secondary users need not occupy all the available channels.

In this paper, we consider un-slotted 1-persistent CSMA as the random multiple access protocols for the secondary users. Since the channel can be occupied by the prime user again in the near future, each secondary user transmits whenever the channel is idle. From [15], we have

$$
R_{i}\left(r_{i}^{n}, \mathbf{r}_{-i}^{n}\right)= \begin{cases}\frac{r_{i}^{n} S^{n}}{\sum_{i} r_{i}^{n}}, & \text { if } G \leq G_{0}, \\ 0, & \text { otherwise, }\end{cases}
$$

where

$$
S^{n}=\frac{G^{n}\left[1+G^{n}+\tau G^{n}\left(1+G^{n}+\tau G^{n} / 2\right)\right] e^{-G^{n}(1+2 \tau)}}{G^{n}(1+2 \tau)-\left(1-e^{-\tau G^{n}}\right)+\left(1+\tau G^{n}\right) e^{-G^{n}(1+\tau)}},
$$


$G^{n}=\sum_{i} r_{i}^{n}$, and $\tau$ is the propagation delay over packet transmission time. When the network payload increases, more collisions happen and consequently the average delay for each packet increases. For some types of payloads like multimedia services, the delayed packets can cause significant QoS loss. In [16], it has been shown that the average delay can be unbounded for a sufficiently large load. Moreover, for cognitive radio, since the prime users can reoccupy the channel in the near future, a certain delay can cause the second user to loss the opportunity for transmission entirely. So we define $G_{0}$ as the maximum network payload. Any network payload larger than $G_{0}$ will cause an unacceptable average delay. As a result, the utility function is zero.

\section{CORRELATED EQUILIBRIUM AND LEARNING}

In this section, we first propose a new solution concept, correlated equilibrium. Then, we investigate a linear programming method to calculate the optimal correlated equilibrium. Finally, we utilize a no-regret algorithm to learn the correlated equilibria in a distributed way.

\section{A. Correlated Equilibrium}

To analyze the outcome of the game, Nash Equilibrium is a well-known concept, which states that in the equilibrium every user will select a utility-maximizing strategy given the strategies of every other user.

Definition 1: Nash Equilibrium $\mathbf{r}_{i}^{*}$ is defined as:

$$
U_{i}\left(\mathbf{r}_{i}^{*}, \mathbf{r}_{-i}\right) \geq U_{i}\left(\mathbf{r}_{i}^{\prime}, \mathbf{r}_{-i}\right), \forall i, \forall \mathbf{r}_{i}^{\prime} \in \Omega_{i},
$$

i.e., given the other users' actions, no user can increase its utility alone by changing its own action.

If a user will follow an action in every possible attainable situation in a game, the action is called pure strategy, in which the probability of using action $\nu_{l}, p\left(r_{i}^{n}=\nu_{l}\right)$, has only one nonzero value 1 for all $l$. In the case of mixed strategies, the user will follow a probability distribution over different possible action, i.e. different rate $l$. In Table I, we illustrate an example of two secondary users with different actions. In Table I (a), we list the utility function for two users taking action 0 and 1 . We can see that when two users take action of 0 , they have the best overall benefit. We can see this action as a cooperative action, or in our case the users transmit less aggressively. But if any user plays more aggressively using action 1 while the other still plays action 0 , the aggressive user has a better utility, but the other user has a lower utility and the overall benefit is reduced. In our case, the aggressive user can achieve a higher rate. However, if both users play aggressively using action 1 , both users obtain the very low utilities. This situation represents the congested network with low throughput of CSMA. In Table I (b), we show two Nash equilibria, where one of the user dominates the other. The dominating user has the utility of 6 and the dominated user has the utility of 3 , which is unfair. In Table I (c), we show the mixed Nash equilibrium where two users have the probability 0.75 for action 0 and 0.25 for action 1 , respectively. The utility for each user is 4.5 .

Next, we study a new concept of correlated equilibrium which is more general than Nash equilibrium and was first
TABLE I: Two secondary users game (a) reward table (upper left); (b) Nash Equilibrium (upper right); (c) Mixed Nash Equilibrium (lower left); (d) Correlated Equilibrium (lower left).

\begin{tabular}{|c|c|c|c|c|c|c|c|}
\hline & \multicolumn{2}{|c|}{0} & 1 & & \multicolumn{2}{|c|}{0} & 1 \\
\hline 0 & \multicolumn{2}{|c|}{$(5,5)$} & $(6,3)$ & 0 & \multicolumn{2}{|c|}{0} & $(0$ or 1$)$ \\
\hline 1 & \multicolumn{2}{|c|}{$(3,6)$} & $(0,0)$ & 1 & \multicolumn{2}{|c|}{$(1$ or 0$)$} & 0 \\
\hline & & 0 & 1 & & & 0 & 1 \\
\hline & 0 & $9 / 16$ & $3 / 16$ & & 0 & 0.6 & 0.2 \\
\hline & 1 & $3 / 16$ & $1 / 16$ & & 1 & 0.2 & 0 \\
\hline
\end{tabular}

proposed by Nobel Prize winner, Robert J. Aumann [18], in 1974. The idea is that a strategy profile is chosen randomly according to a certain distribution. Given the recommended strategy, it is to the players' best interests to conform with this strategy. The distribution is called the correlated equilibrium.

In the rest of this subsection, we assume $N=1$ and we omit the notation $n$. Let $\mathbb{G}=\left\{K,\left(\Omega_{i}\right)_{i \in K},\left(U_{i}\right)_{i \in K}\right\}$ be a finite $K$ user game in strategic form, where $\Omega_{i}$ is the strategy space for user $i$, and $U_{i}$ is the utility function for user $i$. Define $\Omega_{-i}$ as the strategy space for user $i$ 's opponents. Let denote the action for user $i$ and its opponents as $\mathbf{r}_{i}$ and $\mathbf{r}_{-i}$, respectively. Then, the correlated equilibrium is defined as:

Definition 2: A probability distribution $p$ is a correlated strategy of game $\mathbb{G}$, if and only if, for all $i \in K, \mathbf{r}_{i} \in \Omega_{i}$, and $\mathbf{r}_{-i} \in \Omega_{-i}$,

$$
\sum_{\mathbf{r}_{-i} \in \Omega_{-i}} p\left(\mathbf{r}_{i}, \mathbf{r}_{-i}\right)\left[U_{i}\left(\mathbf{r}_{i}^{\prime}, \mathbf{r}_{-i}\right)-U_{i}\left(\mathbf{r}_{i}, \mathbf{r}_{-i}\right)\right] \leq 0, \forall \mathbf{r}_{i}^{\prime} \in \Omega_{i}
$$

By dividing inequality in (8) with $p\left(\mathbf{r}_{i}\right)=$ $\sum_{\mathbf{r}_{-i} \in \Omega_{-i}} p\left(\mathbf{r}_{i}, \mathbf{r}_{-i}\right)$, we have

$$
\sum_{\mathbf{r}_{-i} \in \Omega_{-i}} p\left(\mathbf{r}_{-i} \mid \mathbf{r}_{i}\right)\left[U_{i}\left(\mathbf{r}_{i}^{\prime}, \mathbf{r}_{-i}\right)-U_{i}\left(\mathbf{r}_{i}, \mathbf{r}_{-i}\right)\right] \leq 0, \forall \mathbf{r}_{i}^{\prime} \in \Omega_{i}
$$

The inequality (9) means that when the recommendation to user $i$ is to choose action $\mathbf{r}_{i}$, then choosing action $\mathbf{r}_{i}^{\prime}$ instead of $\mathbf{r}_{i}$ cannot obtain a higher expected payoff to $i$.

We note that the set of correlated equilibria is nonempty, closed and convex in every finite game. Moreover, it may include the distribution that is not in the convex hull of the Nash equilibrium distributions. In fact, every Nash equilibrium is a correlated equilibrium and Nash equilibria correspond to the special case where $p\left(\mathbf{r}_{i}, \mathbf{r}_{-i}\right)$ is a product of each individual user's probability for different actions, i.e., the play of the different players is independent [18]-[20]. In Table I (b) and (c), the Nash equilibria and mixed Nash equilibria are all within the set of correlated equilibria. In Table I (d), we show an example where the correlated equilibrium is outside the convex hull of the Nash equilibrium. Notice that the joint distribution is not the product of two users' probability distributions, i.e., two users' actions are not independent. Moreover, the utility for each user is 4.8 which is higher than that of the mixed strategy. 
TABLE II: The regret-matching learning algorithm

\begin{tabular}{|l|}
\hline Initialize arbitrarily probability for taking action of user $i$, \\
$p_{i}^{1}\left(\mathbf{r}_{i}\right), \forall i \in K$ \\
\hline for $\mathrm{t}=1,2,3, \ldots$ \\
1. Find $D_{i}^{t}\left(\mathbf{r}_{i}, \mathbf{r}_{i}^{\prime}\right)$ as in (12) \\
2. Find average regret $\mathbb{R}_{i}^{t}\left(\mathbf{r}_{i}, \mathbf{r}_{i}^{\prime}\right)$ as in (11) \\
3. Let $\mathbf{r}_{i} \in \Omega_{i}$ be the strategy last chosen by user $i$, \\
i.e. $\mathbf{r}_{i}^{t}=\mathbf{r}_{i}$. Then probability distribution action for \\
next period, $p_{i}^{t+1}$ is defined as \\
$p_{i}^{t+1}\left(\mathbf{r}_{i}^{\prime}\right)=\frac{1}{\mu} \mathbb{R}_{i}^{t}\left(\mathbf{r}_{i}, \mathbf{r}_{i}^{\prime}\right) \quad \forall \mathbf{r}_{i}^{\prime} \neq \mathbf{r}_{i}$ \\
$p_{i}^{t+1}\left(\mathbf{r}_{i}\right)=1-\sum_{\mathbf{r}_{i}^{\prime} \neq \mathbf{r}_{i}} p_{i}^{t+1}\left(\mathbf{r}_{i}^{\prime}\right)$, \\
where $\mu$ is a certain constant that is sufficiently large. \\
\hline
\end{tabular}

\section{B. Linear Programming Solution}

The characterization of the correlated equilibria set illustrates that there are solutions of correlated equilibria that achieve strictly better performance compared to the Nash equilibria in terms of the spectrum utilization efficiency and fairness. However, the correlated equilibrium defines a set of solutions which is better than Nash equilibrium, but it does not tell any more information regarding which correlated equilibrium is most suitable in practice. We propose two refinements. The first one is the maximum sum correlated equilibrium that maximize the sum of utilities of the secondary users. The second one is the maximin fair correlated equilibrium that seeks to improve the worst case situation. The problem can be formulated as a linear programming problem as:

$$
\begin{gathered}
\max _{p} \sum_{i \in K} E_{p}\left(U_{i}\right) \text { or } \max _{p} \min _{i} E_{p}\left(U_{i}\right) \\
\text { s.t. }\left\{\begin{array}{l}
p\left(\mathbf{r}_{i}, \mathbf{r}_{-i}\right)\left[U_{i}\left(\mathbf{r}_{i}^{\prime}, \mathbf{r}_{-i}\right)-U_{i}\left(\mathbf{r}_{i}, \mathbf{r}_{-i}\right)\right] \leq 0, \\
\forall \mathbf{r}_{i}, \mathbf{r}_{i}^{\prime} \in \Omega_{i}, \forall i \in K .
\end{array}\right.
\end{gathered}
$$

where $E_{p}(\cdot)$ is the expectation over $p$. The constraints guarantee the solution is within the correlated equilibrium set.

Notice that the solution in (10) requires all information to be available for optimization. The requirement is not possible for distributed cognitive users. So the solution can be only served as a performance upper bound. In the next subsection, we propose a learning algorithm for the individual secondary user using only local information.

\section{No-Regret Learning Algorithm}

In this subsection, we will exhibit a class of algorithm called regret-matching algorithm [20]. The algorithm was named regret-matching (no-regret) algorithm, because the stationary solution of the learning algorithm exhibits no regret and the play probabilities are proportional to the "regrets" for not having played other actions. In particular, for any two distinct actions $\mathbf{r}_{i} \neq \mathbf{r}_{i}^{\prime}$ in $\Omega_{i}$ and at every time $T$, the regret of user $i$ at time $T$ for not playing $\mathbf{r}_{i}^{\prime}$ is

$$
\mathbb{R}_{i}^{T}\left(\mathbf{r}_{i}, \mathbf{r}_{i}^{\prime}\right):=\max \left\{D_{i}^{T}\left(\mathbf{r}_{i}, \mathbf{r}_{i}^{\prime}\right), 0\right\},
$$

where

$$
D_{i}^{T}\left(\mathbf{r}_{i}, \mathbf{r}_{i}^{\prime}\right)=\frac{1}{T} \sum_{t \leq T}\left(U_{i}^{t}\left(\mathbf{r}_{i}^{\prime}, \mathbf{r}_{-i}\right)-U_{i}^{t}\left(\mathbf{r}_{i}, \mathbf{r}_{-i}\right)\right) .
$$

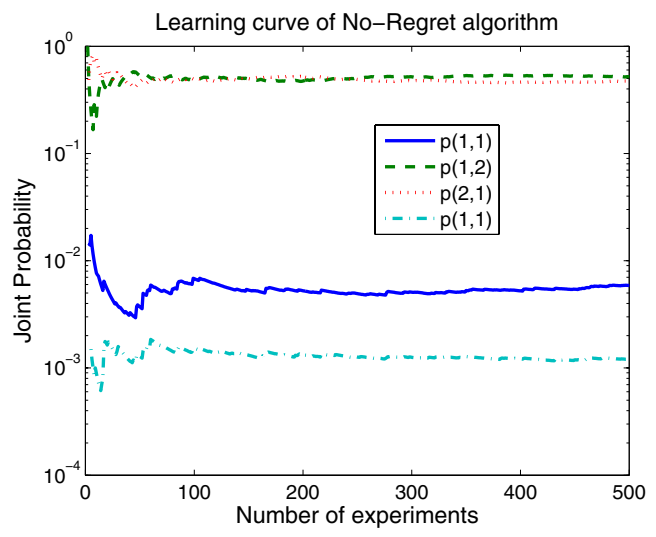

Fig. 2: Learning Curves for No-regret Algorithm

$D_{i}^{T}\left(\mathbf{r}_{i}, \mathbf{r}_{i}^{\prime}\right)$ has the interpretation of average payoff that user $i$ would have obtained, if it had played action $\mathbf{r}_{i}^{\prime}$ every time in the past instead of choosing $r_{i}$. The expression $\mathbb{R}_{i}^{T}\left(\mathbf{r}_{i}, \mathbf{r}_{i}^{\prime}\right)$ can be viewed as a measure of the average regret. The probability $p_{i}\left(\mathbf{r}_{i}\right)$ for user $i$ to take action $\mathbf{r}_{i}$ is a linear function of the regret. The detail regret-matching algorithm is shown in Table II. The complexity of the algorithm is $O(L)$.

For every period $T$, let us define the relative frequency of users' action $\mathbf{r}$ played till $T$ periods of time as follow

$$
z_{T}(\mathbf{r})=\frac{1}{T} \#\left\{t \leq T: \mathbf{r}_{t}=\mathbf{r}\right\}
$$

where $\#(\cdot)$ denotes the number of times the event inside the bracket happens and $\mathbf{r}_{t}$ is all users' action at time $t$. The following theorem guarantees the adaptive learning algorithm shown in Table II has the property that $z_{T}$ converges almost sure to a set of the correlated equilibria.

Theorem 1: [20] If every player plays according to adaptive learning algorithm in Table II, then the empirical distributions of play $z_{T}$ converge almost surely to the set of correlated equilibrium distributions of the game $G$, as $T \rightarrow \infty$.

\section{Simulation Results}

In the simulations, we employ the maximal sum utility function as the objective. In the first simulation, we study the convergence of the no-regret algorithm. In Figure 2, we show a two-user case with actions of $[0.5,1]$. Here $p$ is the joint probability of user' taking action of 1 and 2 . We can see that the learning algorithm converge to the set of the correlated equilibria with about 100 iterations. The fast convergence of the learning algorithm can ensure the second users to obtain the performance gain before the prime users retake the channels.

In Figure 3, we show the different equilibria as a function of $G_{0}$ for three-user game. We show the results of the gain obtained by the greedy user in the Nash equilibrium point (NEP), the gain obtained by the victim of the greedy user in NEP, the learning result, and the optimal correlated equilibrium calculated by linear programming. Here the action space is $[0.1,0.2, \ldots, 1.5]$. When $G_{0}$ is large, there is less penalty for greedy behaviors. So all users tend to transmit as aggressively as possible. This results in the Prison Dilemma [21], where all users suffer. When $G_{0}$ is less than 2.8, the greedy user can have a better performance (NEP best) than that (NEP worst) of 


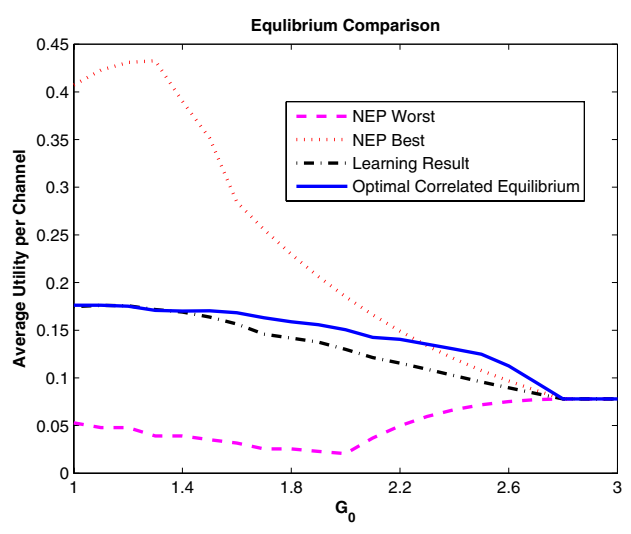

Fig. 3: Utility Function v.s. $G_{0}$, Three Users

the cooperative user. Due to the significant penalty if all users transmit aggressively, the game will not degrade to the Prison Dilemma. However the performances are quite unfair for the greedy users and the cooperative users. All users have the same utility in the correlated equilibrium and learning result. So the fairness is better than the NEP. When $G_{0}$ is from 2.2 to 2.8 , the correlated equilibrium has a better performance even than that of the greedy user (NEP best). When $G_{0}$ is from 1.4 to 2.8, the optimal correlated equilibrium has a better performance than that of the learning result. When $G_{0}$ is sufficiently small, most of the uncooperative strategies are eliminated by significant penalty. Consequently, the learning result has the same performance as that of the optimal correlated equilibrium.

In Figure 4, we show the network performance of the proposed algorithm. For simplicity, we assume the hidden terminal problem [15] has been solved. We show the average user utility per channel as a function of the network density. When the network density is small, the average utility is increasing since there is an increasing number of users to occupy the channel. When the user density is sufficiently large, the utility begins to decrease due to the collisions. The best NEP and worst NEP are different while the correlated equilibrium and learning result achieve almost the same performance as the best NEP and 5\% to $15 \%$ better than the worst NEP.

\section{CONCLuSions}

In this paper, we propose the correlated equilibrium concept for cognitive users to have distributive opportunistic spectrum access. Then, we construct a no-regret learning algorithm to learn the correlated equilibrium. Simulation results show that the correlated equilibrium has a $5 \%$ to $15 \%$ better performance and is more fair, comparable to the NEP.

\section{REFERENCES}

[1] S. Haykin, "Cognitive radio: brain-empowered wireless communications", IEEE Journal on Selected Areas in Communications, vol. 23, no. 2, pp. 201-220, February 2005.

[2] I. F. Akyildiz, W. Y. Lee, M. C. Vuran, and S. Mohanty, "Next generation/dynamic spectrum access/cognitive radio wireless networks: a survey", Computer Networks: The International Journal of Computer and Telecommunications Networking, Volume 50, Issue 13, Pages: 2127 2159, September 2006.

[3] X. Liu and W. Wang, "On the characteristics of spectrum-agile communication networks", The first IEEE International Symposium on New Frontiers in Dynamic Spectrum Access Networks, 2005 (DySPAN 2005), pp. 214-223, Baltimore, MD, November 2005.

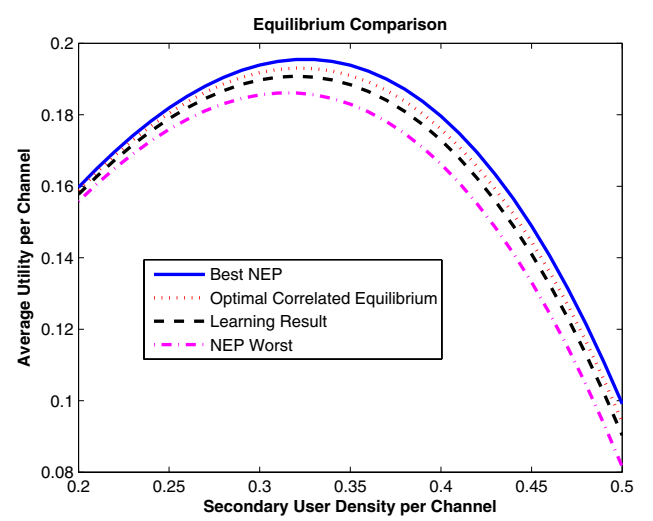

Fig. 4: Network Performances

[4] X. Liu and S. Shankar, "Sensing-based opportunistic channel access", ACM MONET, Volume 11, Issue 4, pp. 577-591 August, 2006.

[5] Q. Zhao, L. Tong, and A. Swami, "Decentralized cognitive MAC for dynamic spectrum access", The first IEEE International Symposium on New Frontiers in Dynamic Spectrum Access Networks, 2005 (DySPAN 2005), pp. 224-232, Baltimore, MD, November 2005.

[6] J. Huang, R. A. Berry, and M. L. Honig, "Spectrum sharing with distributed interference compensation", The first IEEE International Symposium on New Frontiers in Dynamic Spectrum Access Networks, 2005 (DySPAN 2005), pp. 88-93, Baltimore, MD, November 2005.

[7] H. Zheng and L. Cao, "Device-centric spectrum management", The first IEEE International Symposium on New Frontiers in Dynamic Spectrum Access Networks, 2005 (DySPAN 2005), pp. 56-65, Baltimore, MD, November 2005.

[8] C. Saraydar, N. Mandayam, and D. Goodman, "Efficient power control via pricing in wireless data networks," IEEE Transactions on Communications, vol. 50, no. 2, pp. 291-303, Feberary 2002.

[9] Z. Han, Z. Ji, and K. J. Ray Liu, "Fair multiuser channel allocation for OFDMA networks using nash bargaining solutions and coalitions," IEEE Transactions on Communications, vol. 53, no. 8, pp. 1366-1376, 2005.

[10] Z. Han, C. Pandana, and K. J. R. Liu, "A self-learning repeated game framework for optimizating packet forwarding networks", IEEE WCNC, pp. 2131-2136, New Orleans, LA, March 2005.

[11] V. Srivastava, J. Neel, A. MacKenzie, R. Menon, L. A. DaSilva, J. Hicks, J. H. Reed and R. Gilles, "Using game theory to analyze wireless ad hoc networks," IEEE Communications Surveys and Tutorials, vol. 7, no. 4, pp. 46-56, 4th quarter 2005.

[12] R. Etkin, A. Parekh, and D. Tse, "Spectrum sharing for unlicenced bands", The first IEEE International Symposium on New Frontiers in Dynamic Spectrum Access Networks, 2005 (DySPAN 2005), pp. 251-258, Baltimore, MD, November 2005.

[13] N. Nie and C. Comaniciu, "Adaptive channel allocation spectrum etiquette for cognitive radio networks", ACM MONET (Mobile Networks and Applications), special issue on "Reconfigurable Radio Technologies in Support of Ubiquitous Seamless Computing”, to appear, 2006.

[14] J. Neel, J. Reed, R. Gilles. "Game models for cognitive radio algorithm analysis," SDR Forum Technical Conference, pp. 15-18, November 2004.

[15] D. P. Bertsekas and R. G. Gallager, Data network, 2nd edition, PrenticeHall, Inc. 1992.

[16] Y. Yang and T. S. P. Yum, "Delay distributions of slotted ALOHA and CSMA", IEEE Transactions on Communications, vol. 51, no. 11, pp. 1846-1857, Novermber, 2003.

[17] C. Pandana and K. J. Ray Liu, "Near-optimal reinforcement learning framework for energy-aware sensor communications", IEEE Journal on Selected Areas in Communications, vol. 23, pp. 259-268, April 2005.

[18] R. J. Aumann, "Subjectivity and correlation in randomized strategy", Journal of Mathematical Economics, vol. 1, no. 1, pp. 67-96, 1974.

[19] R. J. Aumann, "Correlated equilibrium as an expression of Bayesian rationality”, Econometrica, vol. 55, no. 1, pp. 1 - 18, January 1987.

[20] S. Hart and A. Mas-Colell, "A simple adaptive procedure leading to correlated equilibrium", Econometrica, vol. 68, no. 5, pp. 1127-1150, September 2000.

[21] R. B. Myerson, Game theory: analysis of conflict. 5th edition, Harvard University Press 2002. 